\title{
Découverte de Colpoda steini (Protozoaires, Ciliés, Holotriches) dans les urines d'un malade algérien
}

\author{
par Y. GUY, R. MERAD et K. ADDADI \\ avec la collaboration technique de M. Bahbou \\ (Laboratoire de Parasitologie et de Zoologie Médicale de la Faculté de Médecine \\ et de Pharmacie d'Alger - Laboratoire du Service de Cardiologie du C.H.U.-Mustapha d'Alger)
}

\section{Résumé}

Dans les urines d'un malade présentant une affection cardiaque, les auteurs ont mis en évidence la présence d'un Cilié, Colpoda steini, Maupas, 1888 sous les deux formes végétative et kystique. Après une étude morphologique de ce Protozoaire, il est supposé que le mode de contamination est très vraisemblablement accidentel.

\section{Summary}

In the urine of a patient suffering from heart disease, the A.A. found a Ciliate, Colpoda steini, both in the vegetative and in the cystic forms. After a morphological study of this Protozoa, it is supposed that the way of infection was most likely accidental. 
Le Cilié Colpoda steini Maupas, 1888, n'a été trouvé à notre connaissance qu'une seule fois chez l'homme, et encore s'agissait-il de ses fèces.

Le porteur dont le cas est relaté ici est un homme de 57 ans, travaillant dans une confiserie et entré le 16 mai au Service de Cardiologie du C.H.U.-Mustapha d'Alger où l'on posait le diagnostic d'arythmie complète par fibrillation auriculaire Rien de notable n'est à rapporter dans ses antécédents si ce n'est une crise d'uricémie. Un traitement à base d'anticoagulants fut institué et l'on retrouve aussi la notion d'une thérapeutique à base de colchicine lors de l'accès goutteux.

Un bilan général était établi et l'on demandait plus particulièrement un test d'Addis.

Cet examen montrait des urines claires et limpides et un culot sans hématies ni leucocytes. Mais l'on devait constater par contre, la présence d'un grand nombre de Ciliés, très mobiles, nettement visibles au grossissement $n^{\circ} 10$, plus difficiles à observer au $\mathrm{n}^{\circ} 40$ en raison de la rapidité de leurs mouvements.

Notre surprise devant cette découverte inattendue nous amena à emporter au Laboratoire de Parasitologie de la Faculté de Médecine un flacon contenant les urines émises le matin même à 8 heures. Mais si celles-ci devaient nous permettre la diagnose du Cilié, nous ne devions plus le retrouver par la suite. Le lendemain 18 mai en effet, les urines de 24 heures étaient claires et libres de tout élément. Le 19 mai, il en était de même pour les urines de la première miction. Le 20 mai par contre, les urines de 24 heures contenaient d'assez nombreux leucocytes, quelques hématies et des cylindres granuleux et hyalins ainsi que des cellules épithéliales, mais toujours pas de Ciliés.

En conséquence, notre première impression fut qu'il devait s'agir d'une souillure du flacon ayant servi à recueillir les urines. Nous avons donc contrôlé le mode de nettoyage de ces flacons. Les urines sont récoltées dans des flacons de sérum lavés avec un savon détergent et rincés à l'eau du robinet puis séchés. Tous les flacons sont lavés de la même façon, par le même employé et dans la même eau. Chaque malade urine directement dans son flacon. Les tubes servant aux manipulations ultérieures sont lavés et stérilisés.

Or, le jour où les urines qui nous occupent furent prélevées, six autres tests d'Addis furent effectués sur des malades du même service et les urines recueillies dans un même lot de flacons. Mais, seul notre patient devait présenter des urines bourrées de Ciliés.

A notre corps défendant, pourrait-on dire, nous devions écarter l'hypothèse pourtant la plus vraisemblable d'une souillure accidentelle et les investigations se poursuivirent donc.

Un second examen direct fut effectué l'après-midi même au Laboratoire de la Faculté sur le culot urinaire de centrifugation.

Il fut possible d'étudier au faible grossissement la mobilité de ces Ciliés qui semblait, d'ailleurs, diminuer depuis le matin. Les déplacements s'effectuaient grâce aux cils mais aussi par des contractions et des élongations du corps et le Protozoaire chan- 
geait brusquement de direction. L'aspect ovoïde (plutôt qu'en haricot) permettait de constater dans certaines positions la présence d'une véritable protubérance au-dessus du péristome.

Près de ces formes végétatives, on notait aussi la présence de kystes à différents stades.

Il ne restait alors qu'une très faible quantité du culot urinaire mais il est possible de signaler qu'une partie de celui-ci a été conservé dans de l'eau formolée à $5 \%$ et les Ciliés sont encore bien conservés quelques six mois plus tard.

Le lendemain, après un contrôle des urines où les Ciliés étaient totalement absents, ces urines fraîches furent mélangées au culot de la veille. Vingt-quatre heures après, les Protozoaires y pullulaient comme si les urines constituaient un excellent milieu de culture.

Mais il faut souligner ici qu'un laps de temps de plus de vingt heures avait été nécessaire pour retrouver les Ciliés en nombre notable. La constatation de ce délai semble donc constituer un deuxième argument en défaveur d'une souillure externe des urines du premier jour, celles-ci ayant été examinées pour la première fois dans l'heure suivant leur émission: il semble donc que les Protozoaires existaient déjà dans les urines. Il fut possible de confirmer par l'expérimentation qu'un délai d'une heure est nettement insuffisant pour constater leur présence. En effet, dans de l'eau croupie contenant soit du cresson, soit de l'herbe et du gazon, il fut donné de vérifier qu'il fallait en moyenne à la température du laboratoire plus de quatre jours pour voir apparaître quelques rares Ciliés et plus de huit jours pour noter une pullulation conséquente. Les Ciliés alors retrouvés appartenaient, par ailleurs, aussi au genre Colpoda.

Trois colorations simples furent employées pour l'identification du Cilié : rouge neutre à $1 \%$ et éosine à $1 \%$ (en solution aqueuse et après fixation à l'eau formolée à $5 \%$ ) et teinture de Merthiolate directement. De ces trois procédés, nous avons retenu la coloration à l'éosine qui donnait les meilleures préparations, le rouge neutre, trop pénétrant, tendant à opacifier ces Ciliés et le Merthiolate les déformant par contraction.

On employait aussi une coloration élective à l'Hématoxyline ferrique après fixation au sublimé de Schaudinn.

Nous avons remarqué que pour fixer l'étalement sur les lames un peu de salive donnait de bien meilleurs résultats que le sérum ou l'albumine de Mayer.

La coloration à l'éosine permet d'étudier surtout la morphologie cytoplasmique de la forme végétative.

Les mesures sont rapportées dans le tableau ci-dessous. Elles concernent la plus grande longueur et la plus grande largeur pour les formes végétatives et le plus grand diamètre et le plus petit diamètre pour les formes kystiques.

La coloration à l'éosine des formes végétatives fait bien apparaître la membrane cytoplasmique très fine et recouverte de cils. Elle présente cinq encoches au niveau du 
promontoire. Le péristome s'ouvre au-dessous et la touffe des cils adoraux, souvent bien visible, prend naissance à sa partie inférieure et non pas à son pourtour ; ces cils sortent du péristome en une touffe assez fournie. Le cytostome se trouve tout près du noyau. Tout près de lui également et en arrière, on note la présence constante d'une grosse vacuole claire qui ne prend pas les colorants, parmi d'autres, sombres et colorés dans le cytoplasme granuleux. On retrouve là la notion classique de vacuole pulsatile et de vacuole digestive. Le noyau, souvent très peu visib!e, apparaît en surimpression.

\section{Tableau 1}

Mesures des formes végétatives et kystiques (Eosine à 1 \%)

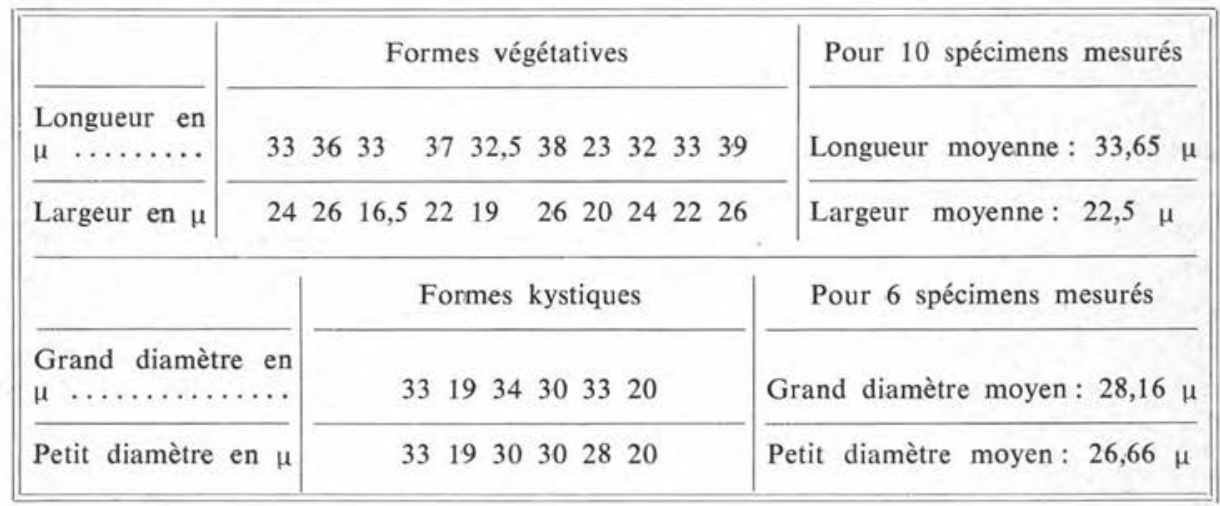

C'est la conformation particulière du promontoire qui nous a fait poser la diagnose de Colpoda steini Maupas, 1888. Il faut bien souligner cependant que le chiffre de cinq lobes est ceiui que nous avons très généralement retrouvé et ce n'est qu'après de nombreuses hésitations que nous avons pu compter peut-être six lobes dans de rares occasions. Si nous nous sommes résolus cependant à ne pas créer une $s p$. nov. mais à identifier ce Cilié à $C$. steini, c'est parce qu'il en est de toute façon très proche. Il faut souligner pourtant que Wenyon dit (4) que ce Protozoaire présente six à sept lobes mais, par contre, la figure qu'il donne à ce sujet n'en montre que cinq. De plus, suivant la coloration, la longueur moyenne du Cilié (of. Tableaux 1 et 2) dont il est question ici est de : 33,65 ou $36,1 \mu$. Wenyon donne comme mesure pour $C$. steini : 23 à $48 \mu$, alors que $C$. maupasi a de 45 à $70 \mu$ (il n'a d'ailleurs jamais été signalé chez l'homme ; il comporte aussi six à sept lobes) et $C$. cucullus, de 40 à $100 \mu$ (et neuf à dix lobes). Enfin, si le macronucléus n'est pas toujours ovoïde comme chez C. steini classique, son nucléole, par contre, est toujours bien visible et arrondi et le micronucléus est toujours bien individualisé hors de lui et souvent ovoïde. La ressemblance de notre Cilié avec $C$. steini, bien qu'imparfaite, ne nous semble pas justifier la création d'une nouvelle espèce. 
Si la photographie $\mathrm{n}^{\circ} 1$ ne permet que de deviner le noyau et le péristome, les cils et la touffe adorale sont assez nettement individualisés; la région anale se devine également. Le colorant comme pour les deux photographies suivantes était l'éosine à $1 \%$. La photographie $\mathrm{n}^{\circ} 2$ montre mieux le péristome et le cytostome. La photographie $n^{\circ} 3$ enfin permet de voir plus nettement le noyau, la vacuole et surtout les lobes avec leurs encoches sur le promontoire.

Certains champs de préparation faisaient apparaître des images de division binaire (photographies $\mathrm{n}^{\circ} 4$ et 5 ; hématoxyline ferrique) et il semble bien que la photographie $\mathrm{n}^{\circ} 6$ représente le phénomène de reproduction sexuée, la conjugaison, si remarquablement décrite par Maupas. Notons enfin (photographie $n^{\circ} 7$; hématoxyline) une phase précoce de l'amitose chez une forme végétative.

Il a été possible de préciser la morphologie de l'appareil nucléaire grâce à la coloration à l'hématoxyline ferrique. On retrouve le macronucléus, tantôt ovoïde, tantôt arrondi, qui présente dans cette espèce un gros caryosome central arrondi, et, tout contre lui, le micronucléus où l'on devine aussi un caryosome (photographies 8,9 et 10$)$.

Des kystes furent également étudiés. Plusieurs stades de l'enkystement ont été vus. La vacuole pulsatile existe au début puis disparaît ainsi que les cils et le cytopharynx (photographie 11, hématoxyline ferrique; 12 et 13 : éosine).

Les mensurations effectuées sur les préparations colorées à l'hématoxyline ferrique ont donné des chiffres légèrement supérieurs pour les formes végétatives à ceux relevés sur les préparations à l'éosine. La fixation vitale au sublimé de Schaudinn respecte probablement davantage la taille des Protozoaires et ces derniers chiffres trouvés correspondent mieux à ceux donnés par Wenyon (23 à $48 \mu)$. On peut constater également dans ce tableau 2 que la mensuration des kystes fait mieux ressortir l'infériorité de leur taille par rapport à celle des formes végétatives (ces mesures sont également inférieures à celles correspondantes du tableau 1).

\section{Tableau 2}

Mesures des formes végétatives et kystiques (Hématoxyline ferrique)

\begin{tabular}{|c|c|c|}
\hline & Formes végétatives & Pour 10 spécimens mesurés \\
\hline $\begin{array}{l}\text { Longueur en } \\
\mu \quad \ldots \ldots \ldots\end{array}$ & $\begin{array}{llllllllll}39 & 41 & 29 & 38 & 39 & 37 & 31 & 41 & 36 & 30\end{array}$ & Longueur moyenne: $36,1 \mu$ \\
\hline \multirow[t]{2}{*}{ Largeur en $\mu$} & $\begin{array}{llllllllll}28 & 28 & 23 & 32 & 28 & 28 & 23 & 28 & 30 & 24\end{array}$ & Largeur moyenne: $27,2 \mu$ \\
\hline & Formes kystiques & Pour 6 spécimens mesurés \\
\hline $\begin{array}{l}\text { Grand diamètre en } \\
\mu \ldots \ldots \ldots \ldots \ldots \ldots\end{array}$ & $\begin{array}{llllll}28 & 17 & 28 & 30 & 31 & 19\end{array}$ & Grand diamètre moyen : $25,5 \mu$ \\
\hline Petit diamètre en $\mu$ & $\begin{array}{llllll}26 & 14 & 28 & 28 & 26 & 17\end{array}$ & Petit diamètre moyen : $23,16 \mu$ \\
\hline
\end{tabular}


Quant au mode de contamination du malade, il n'a pu être élucidé. Il est invraisemblable que ce porteur ait été infecté par voie digestive et il semble bien que la seule voie urinaire rétrograde et accidentelle ait pu servir de porte d'entrée. Notons à ce sujet que le patient, d'un certain âge, est un Musulman pratiquant et qu'il accomplit donc chez lui ou sur le lieu de son travail sa toilette rituelle et quotidienne avec une eau qui peut être souillée et à la base de sa contamination.

On ne sait pas non plus si ce malade a présenté auparavant des Ciliés dans ses urines mais il ne rapporte en tout cas aucun épisode urinaire.

Ce Cilié, cité par Brumpt, Neveu-Lemaire et Wenyon, a été décrit de façon exhaustive par ce dernier.

L'existence de $C$. steini dans des fèces humaines a été signalée une seule fois à notre connaissance en Russie par Yakimov et Kolparoff en 1921 (4) (1923 pour Brumpt et pour Neveu-Lemaire) après culture aseptique de selles.

Il semble que la présence de ce Cilié dan.3 les urines de l'homme n'ait pas encore été signalée. On ne peut s'en étonner car sa brève apparition dans les urines de notre malade aurait pu facilement passer inaperçue si un test urinaire n'avait été demandé. Par ailleurs, on ne peut pas parler de parasitose vraie mais il faut beaucoup plus vraisemblablement admettre un simple transport accidentel n'entraînant pas de trouble pathologique.

\section{Références}

1. Brumpt E, 1949. - Précis de Parasitologie, 6" éd., Masson et $\mathrm{C}^{\mathrm{ie}}$, Paris.

2. Hutner S. H., et Lwoff A., 1955. - Biochemystry and Physiology of Protozoa. Acad. Press. inc. Publish., New York.

3. Neveu-Lemaire M., 1943. - Traité de Protozoologie médicale et vétérinaire. Vigot Frères éd., Paris.

4. Wenyon C.-M., 1926. - Protozoology. Baillıere Tindall et Cox éd., Londres. 


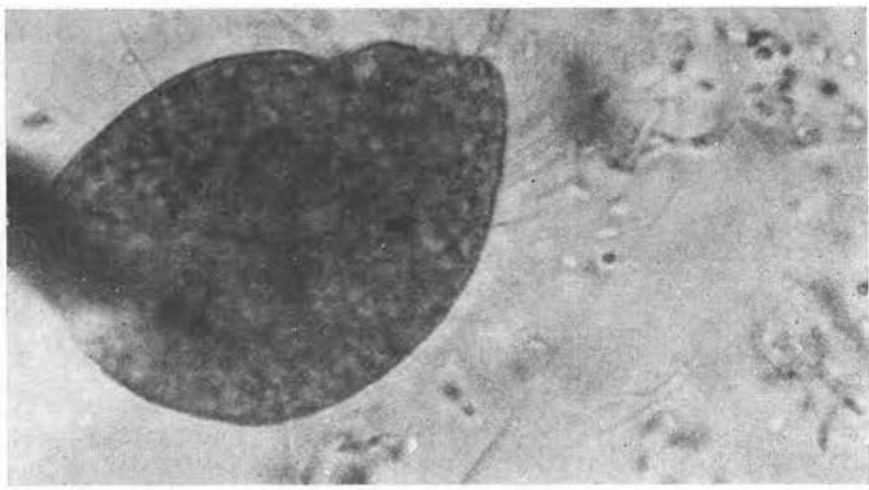

Fig. 1. - Forme végétative. Noter les cils vibratils et la touffe de cils adoraux

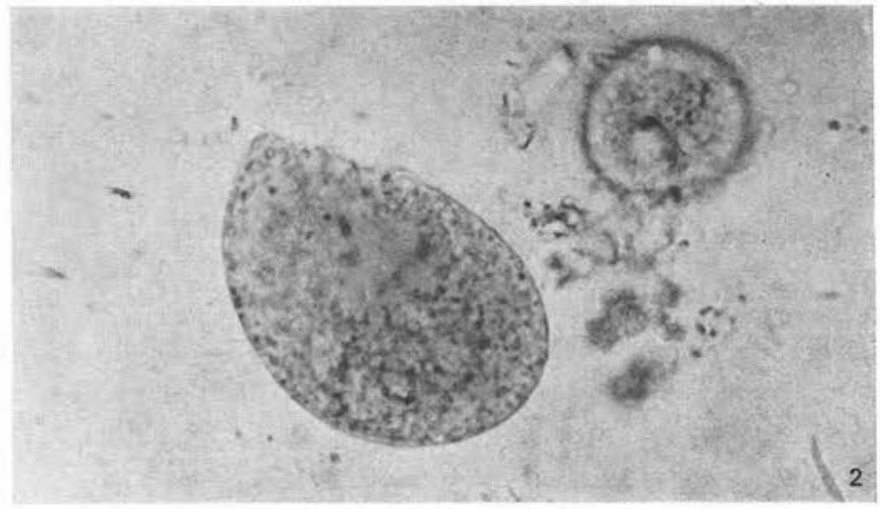

Fig, 2. - Forme végétative. Noter le péristome et le cysostome

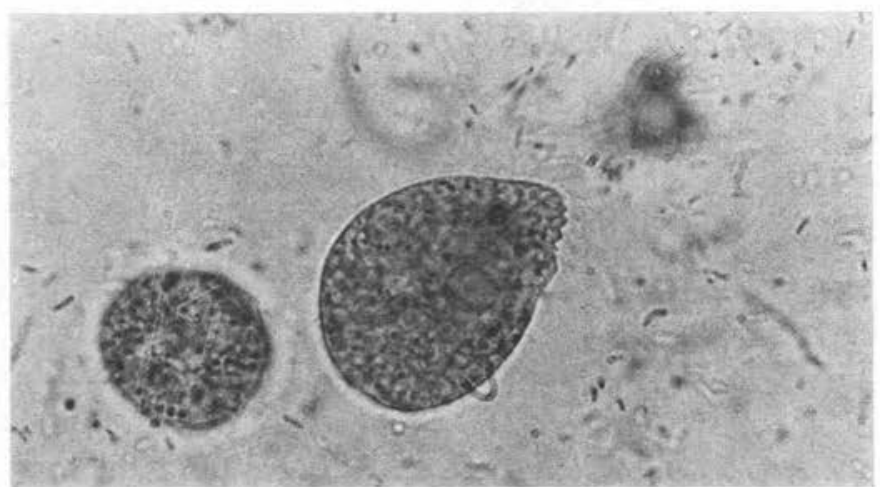

FIG. 3. - Forme végétative. Noter le noyau et les lobes du promontoire 

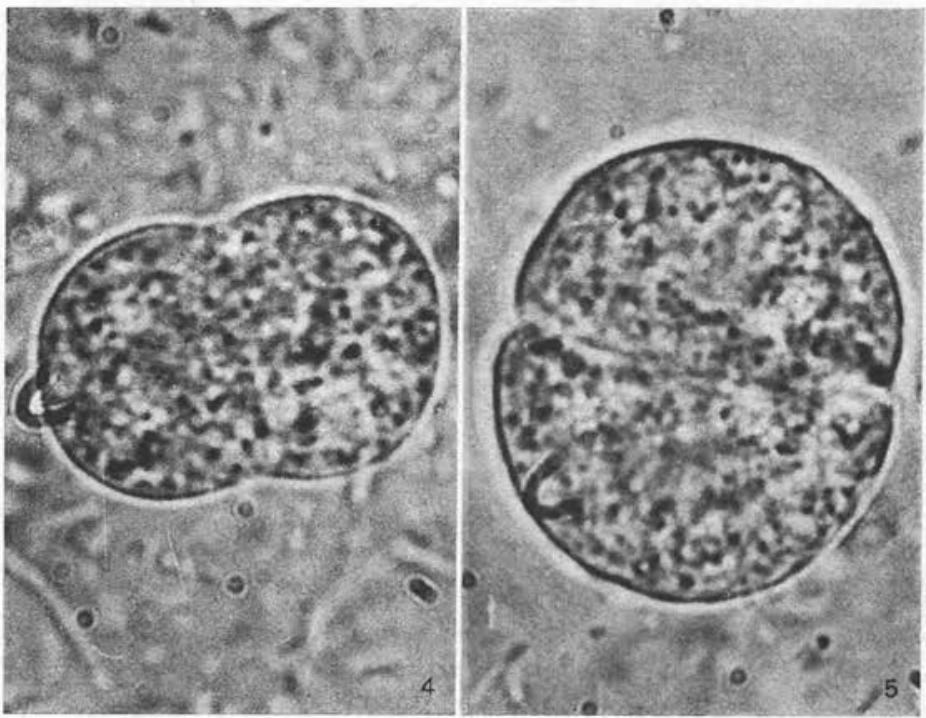

FIG. 4 et 5. - Formes kystiques. Division binaire
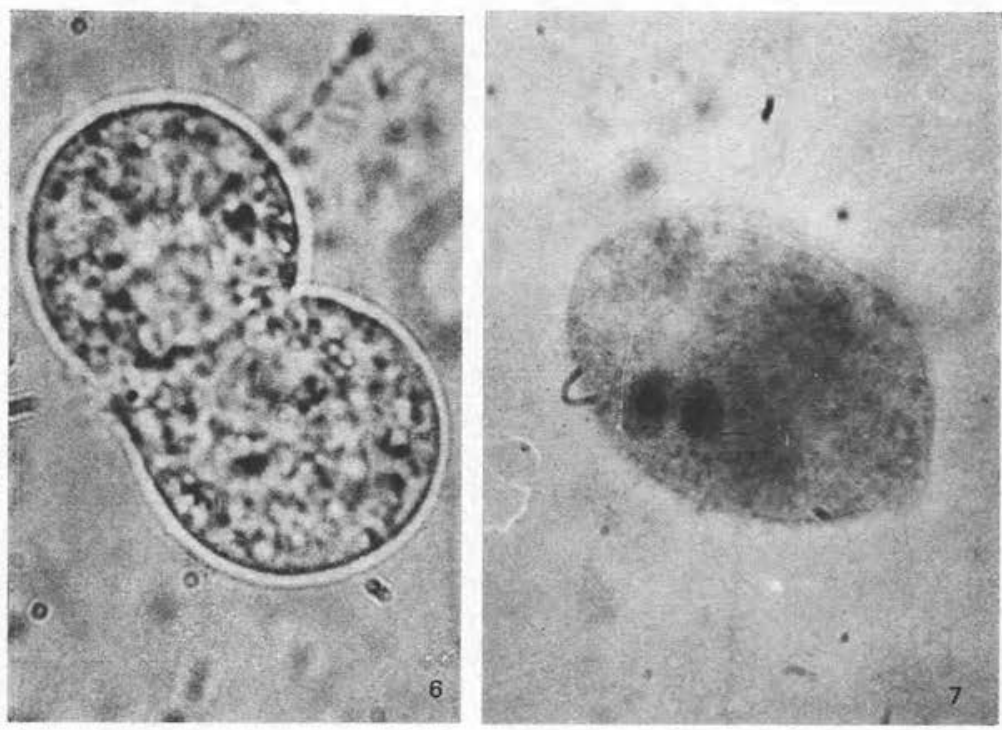

Fig. 6. - Formes végétatives. Phé- FIG. 7. - Forme végétative. Division nomène de conjugaison précoce du noyau 


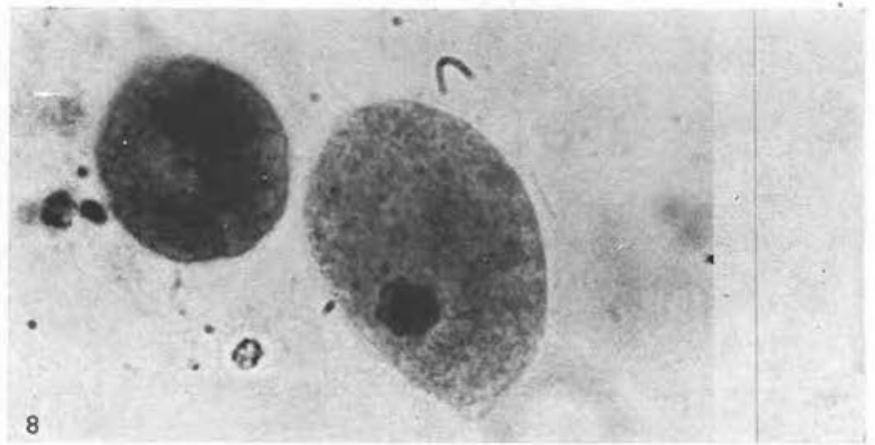

FIG. 8

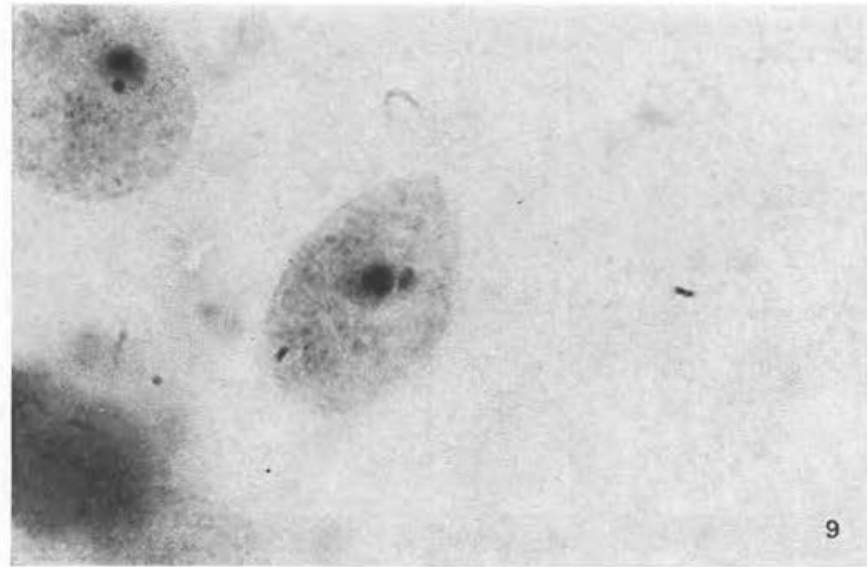

FIG. 9

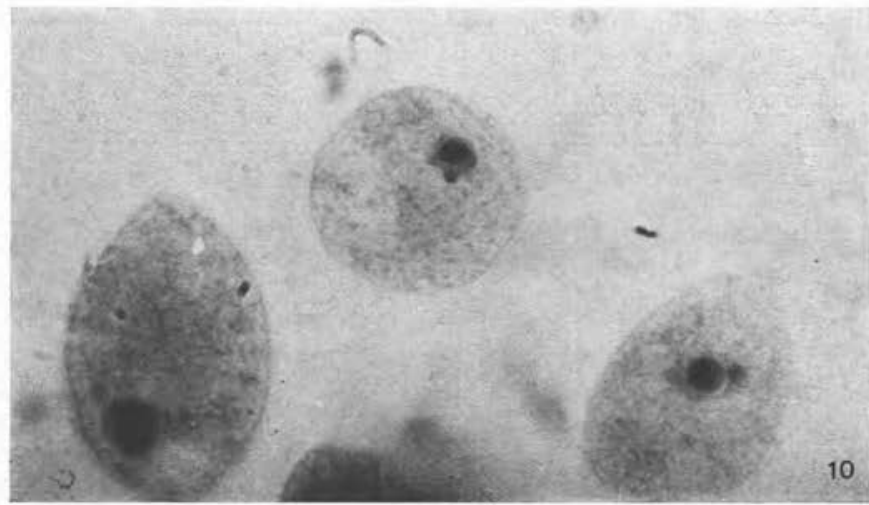

FIG. 10

FIG. 8, 9 et 10. - Formes végétatives. Noter le macronucléus, arrondi ou ovalaire, avec son gros caryosome et le micronucléus placé tout contre lui 


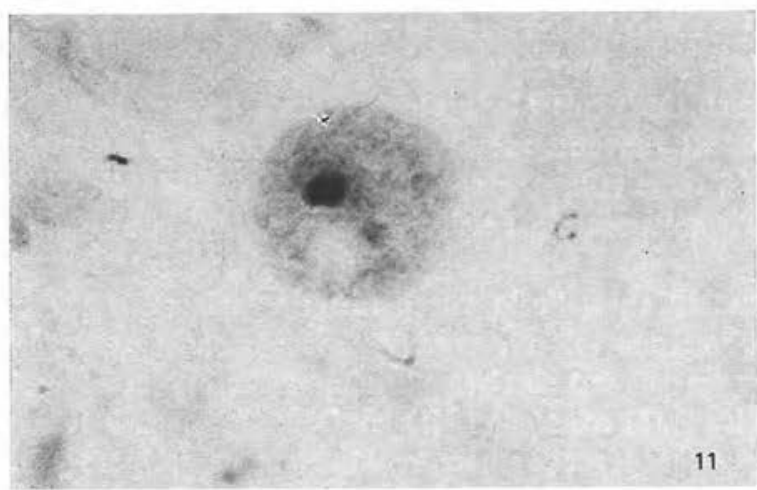

FIG. 11. - Forme kystique. Noter la vacuole pulsatile qui n'a pas encore disparu

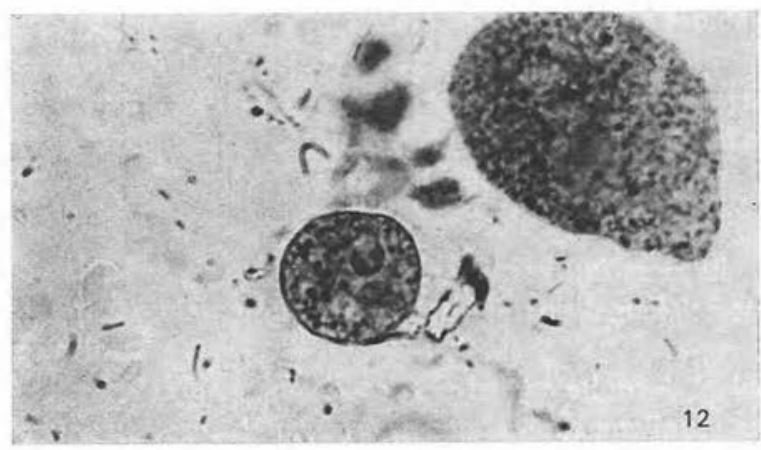

FIG. 12

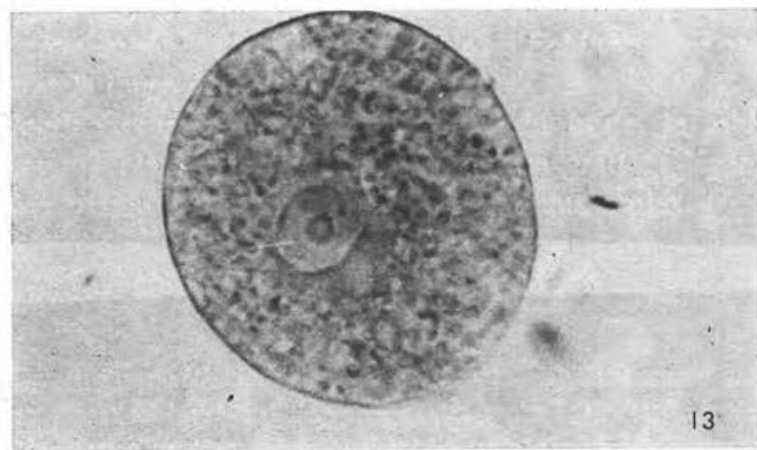

FIG. 13

FIG. 12 et 13. - Formes kystiques. Noter la disparition des cils et de la vacuole 